\title{
Detalles sobre la ubicación y la orientación de los Altares $G$ del Parque Arqueológico de Copán, Honduras
}

Vito Véliz,

María Cristina Pineda de Carías y

Ricardo Agurcia Fasquelle

\section{Resumen}

El objetivo de este trabajo ha sido determinar si los tres altares G1, G2 y G3, localizados en el lado este de la Gran Plaza del Parque Arqueológico de Copán entre las Estelas $\mathrm{F} \mathrm{y} \mathrm{H}$, tienen actualmente la misma orientación longitudinal con que originalmente los colocaron los mayas. Después de una revisión cuidadosa y comparativa de las diferentes anotaciones, referencias y descripciones realizadas por diferentes exploradores, particularmente arqueólogos, que a lo largo del tiempo han incluido en sus trabajos de campo e investigaciones a los altares $G$, y después de realizar observaciones y análisis arqueoastronómicos de las puestas del Sol, situados en medio de los altares $\mathrm{G}(\mathrm{G} 1, \mathrm{G} 2, \mathrm{G} 3)$, hemos llegado a la conclusión de que lo más probable es que la orientación actual de los altares $\mathrm{G}$ (G1, G2 y G3) es la orientación original con que el Décimo Sexto Gobernante de Copán los colocó, marcando con las posiciones de los ejes longitudinales, las direcciones norte-sur, extendida hasta las Estelas $\mathrm{F}$ y $\mathrm{H}$, y la dirección este-oeste, extendida ésta última hasta tomar en cuenta a la Estela 4.

Palabras Clave: Mayas, Copán, Altares $G(G 1, G 2, G 3)$, orientaciones, Arqueoastronomía.

\section{Abstract}

The goal of this research has been to determine whether the three altars; G1, G2 and G3, located on the eastern side of the Great Plaza of the Copan Archaeological Park, between stelae $\mathrm{F}$ and $\mathrm{H}$, currently have the same longitudinal orientation set 
by the ancient Maya. After a careful and comparative revision of various notes, references and descriptions made by different observers, particularly archaeologists who throughout time have included altars $G$ as part of their enquiries and field work, and after carrying out observations and archaeoastronomical analyses of sunsets, in the middle of altars $G(G 1, G 2, G 3)$, we have concluded that it is most likely that the current orientation of the $G$ altars $(G 1, G 2$, and $G 3)$ is the original orientation set by the Sixteenth Ruler of Copan, marking with the position of the longitudinal axes the north-south directions, extended to the Stelae $\mathrm{F}$ and $\mathrm{H}$, and the east-west direction, this one encompassing Stela 4.

Keywords: Mayas, Copan, Altars G (G1, G2, G3), orientations, Archaeoastronomy.

Vito Veliz. Director, Instituto de Arqueoastronomía y Patrimonio Cultural y Natural (IARPACUNA), Facultad de Ciencias Espaciales, Universidad Nacional Autónoma de Honduras. Email: gymacay@hotmail.com.

María Cristina Pineda de Carías. Instituto de Arqueoastronomía y Patrimonio Cultural (IARPACUNA), Facultad de Ciencias Espaciales (FACES), Universidad Nacional Autónoma de Honduras (UNAH). Email: mcpinedacarias@gmail.com.

Ricardo Agurcia Fasquelle. Coordinador de Investigaciones, Instituto Arqueoa stronomía y Patrimonio Cultural y Natural (IARPACUNA), Facultad de Ciencias Espaciales, Universidad Nacional Autónoma de Honduras. Email:raf@asociacioncopan.org. 


\section{INTRODUCCIÓN}

En el Patio del Sol, sección norte de la Plaza Principal del Parque Arqueológico de Copán, en su lado este y entre las estelas $F$ y $\mathrm{H}$, aparecen tres esculturas longitudinales que se conocen como los Altares $G(G 1, G 2, G 3)$. Estos altares representan serpientes bicéfalas emplumadas. Dos de los altares son más pequeños que el tercero. En los dos altares más pequeños, en el centro, a cada lado y por debajo del cuerpo del monstruo, aparece un corte sobre el cual existe una inscripción corta; en el altar más grande, a cada uno de sus lados, aparece una inscripción más larga. Nosotros hemos estudiado la ubicación y orientación de estos altares, conforme lo han reportado desde los primeros exploradores hasta su actual localización, presentando aquí nuestros resultados.

La referencia más temprana sobre estos altares $G$ la encontramos en 1841, en el libro de Stephens sobre sus Incidentes de Viaje en Centroamérica, Chiapas y Yucatán (1969, pág. 152). Aquí él habla de un bulto de escultura caída y menciona lo que él llama un altar, al cual le asigna la letra R. Según Stephens este altar estaba a unos 50 pies ( 15 metros) al sur del monumento T. Hoy día este monumento T se conoce como la Estela I y se supone que $R$ es uno de los altares $G$.

Maudslay (1898-1902) fue el siguiente explorador quien menciona estos altares y quien les asignó la letra $\mathrm{G}$, con números del 1 al 3 , nomenclatura que se conserva aún hoy día. En su libro Biologia Centrali-Americana, Maudslay presenta dos fotografías de los altares G, que nosotros incluimos en las Figuras 1 y 2. 


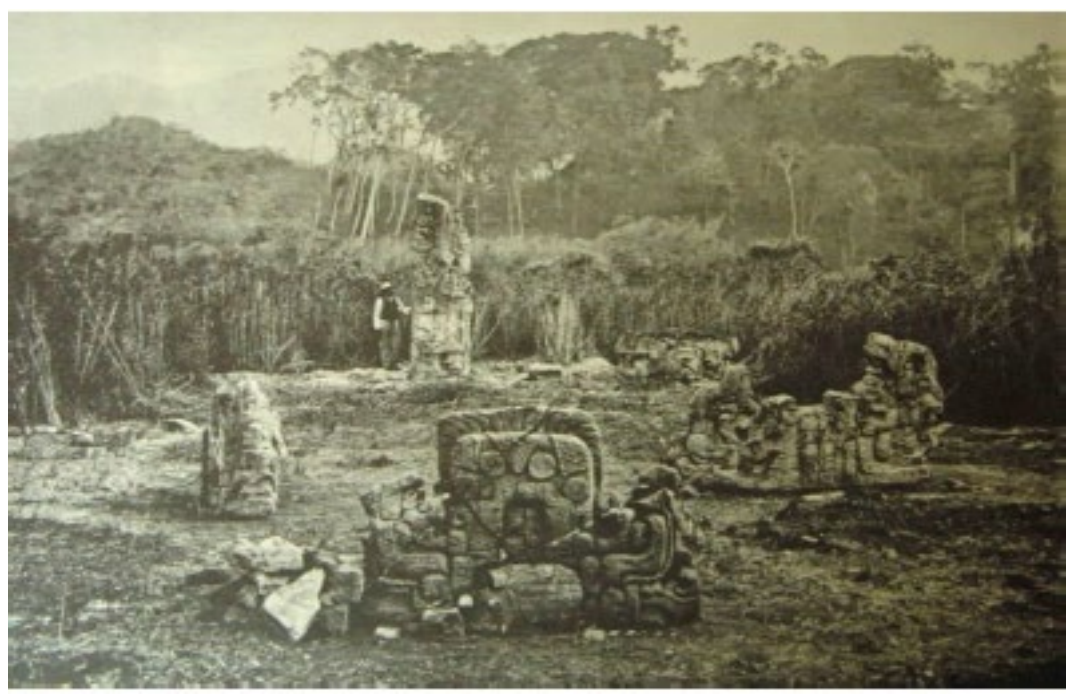

Figura 1. Fotografía de los altares $\mathrm{G}$, vistos en dirección sur. Al fondo, detrás de la Estela $\mathrm{H}$ que mira hacia el oeste, aparece una persona parada. Al frente, en el centro y al lado derecho, aparecen respectivamente los Altares G3 y G1, ambos orientados en dirección este-oeste. A la izquierda, orientado en dirección norte-sur, aparece el Altar G2. Fuente: Maudslay 1898-1902: Plate 117.

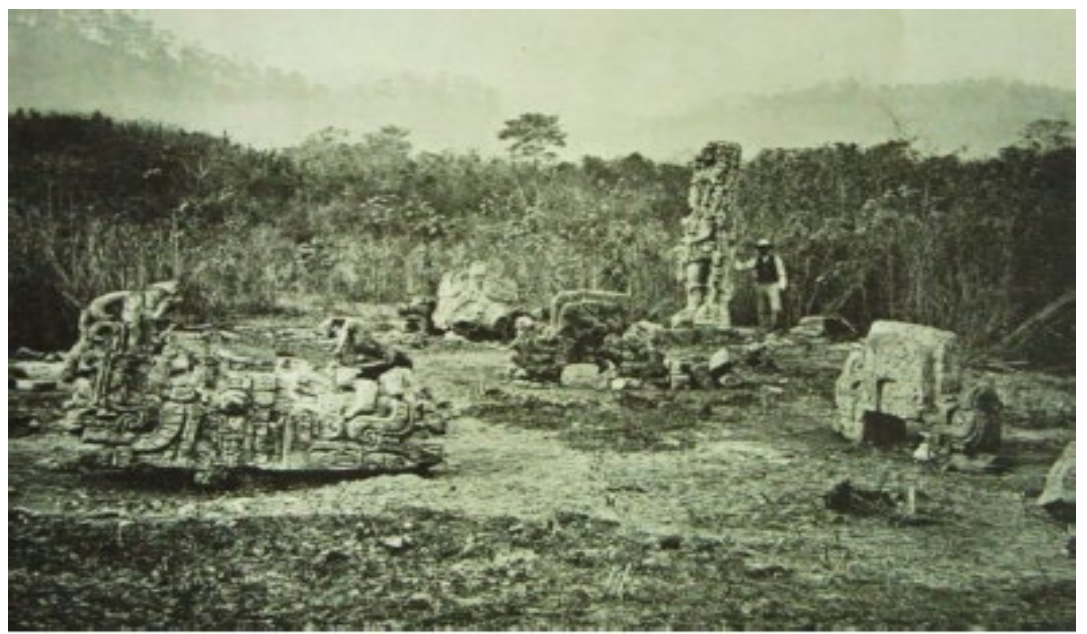

Figura 2. Fotografía de los altares $\mathrm{G}$, vistos en dirección norte. Al fondo, parado detrás de la Estela F que mira hacia el oeste, aparece una persona. Al frente, hacia la izquierda y en el centro, orientados en dirección este-oeste, aparecen respectivamente los altares G1 y G3. Al lado derecho, orientado en dirección norte-sur, aparece el Altar G2.

Fuente: Maudslay 1898-1902: Plate 116. 
Ya en el siglo XX Baudez (1994, pág. 55), en su descripción de las Esculturas de Copán, hace referencia a que en 1885 Maudslay vio juntos los altares $\mathrm{G}$ en la Plaza Principal entre las estelas $\mathrm{H}$ y $\mathrm{F}$, donde permanecen. También menciona que su localización sugiere que ellos fueron colocados como una contraparte de la Estela 4 (CPN 43). Baudez describe que todos los altares miran hacia el lado oeste de la Plaza completando el alineamiento de los monumentos del este: Estela H (CPN16), Altar G1 (CPN 13), Altar G2 (CPN 14), Altar G3 (CPN 15) y Estela F (CPN11). Aclara sin embargo que ellos pueden ser esculturas arquitectónicas originalmente parte de algún edificio. Para la fecha de dedicatoria, haciendo referencia a un trabajo realizado con Riese, Baudez expresa que (1994, pág. 55) estas tres esculturas deben ser consideradas como un solo texto que debe ser leído secuencialmente, empezando con el Altar G3 con fecha dedicatoria de 9.17.0.0.013 Ahau 18 Cumkú; seguida del Altar G2 con fecha dedicatoria de 9.18.5.0.0 4 Ahau 13 Ceh; y terminando con el Altar G1 con fecha dedicatoria de 9.18.10.0.0 10 Ahau 8 Zac. En las Figuras 3 y 4 se muestran los detalles de los altares $G$ que aparecen en el libro de Baudez. 


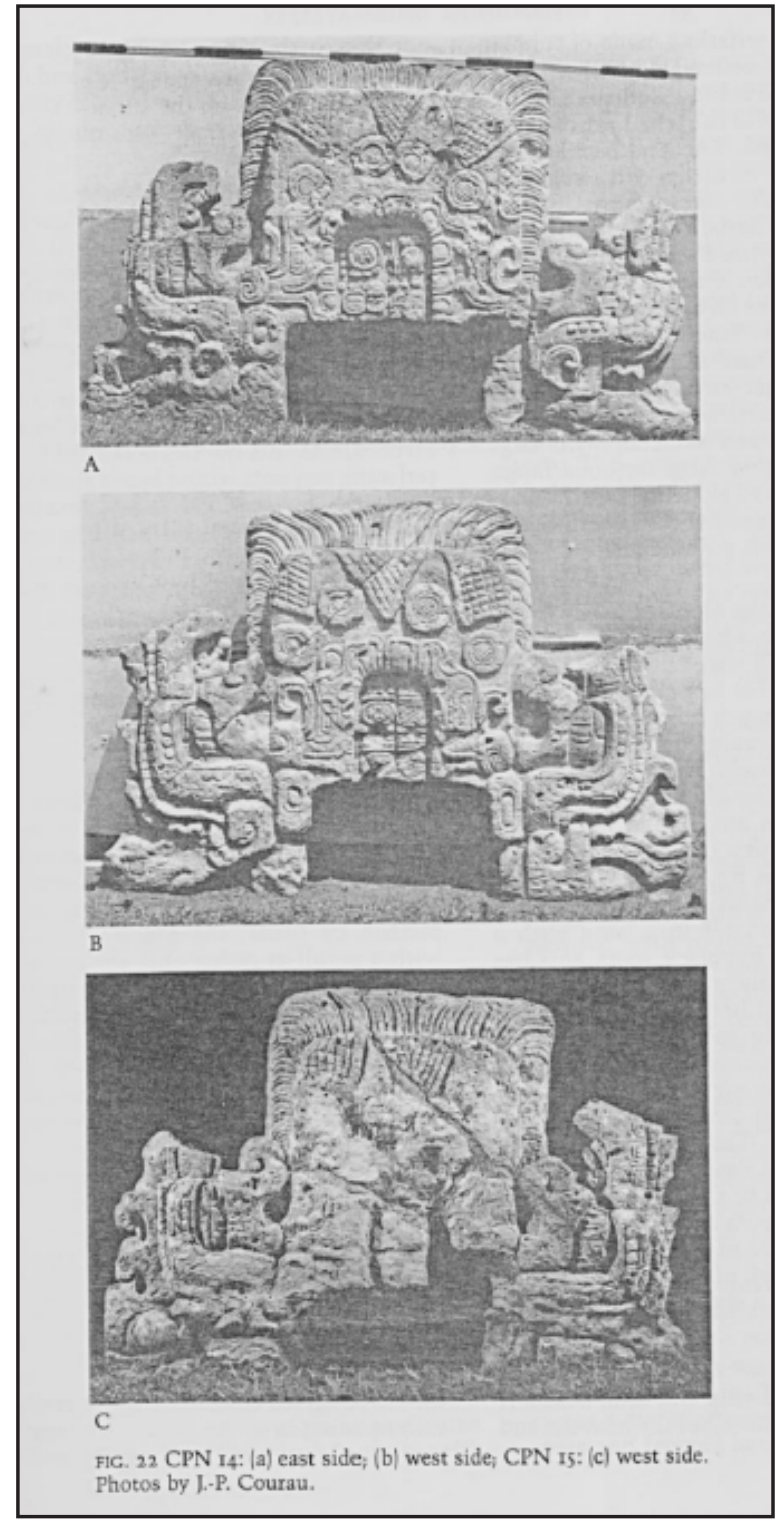

Figura 3. CPN 14 (Altar G2) (a) lado este; b) lado oeste. CPN 15 (Altar G3): c) lado oeste. Fuente: Baudez (1994, pág. 57; Fotos: J.-P. Courau). 


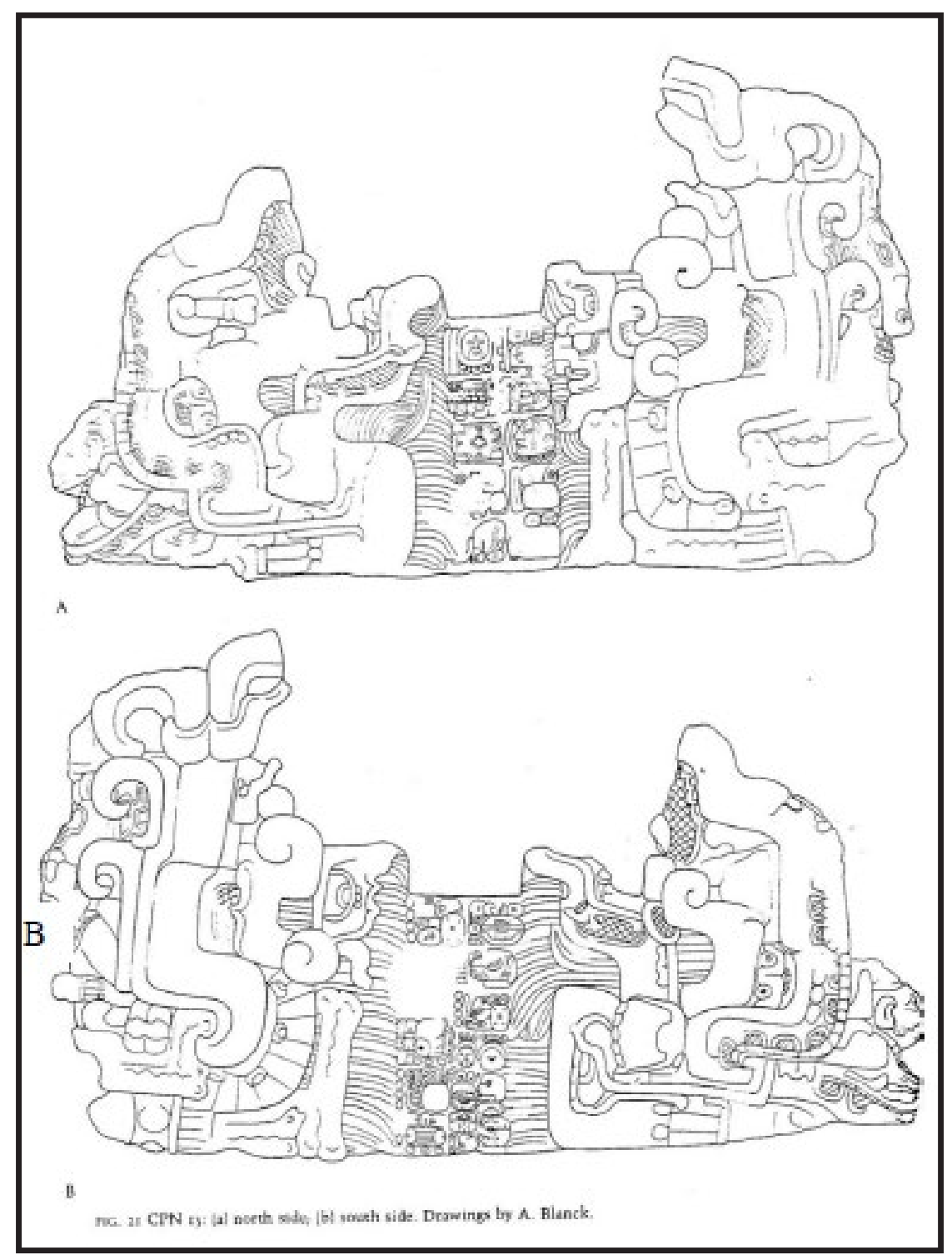

Figura 4. CPN 13 (Altar G1). a) Lado Norte; b) Lado sur.

Fuente: (1994, pág. 56; con Dibujos de A. Blanck). 
En su libro The Code of Kings, Schele y Mathews (1998, pág. 170), refiriéndose a los altares de serpientes de la Gran Plaza de Copán, dicen que Yax-Pasaj, el último rey de la dinastía de Yax-Kuk' Mo, agregó su propia firma al programa de las estelas de Waxaklajún Ubah'Káwil, colocando los altares G. En una fotografía que nosotros incluimos en la Figura 5, ellos muestran la disposición de estos altares (1998, pág. 170). Schele y Freidel (1990, pág. 337 Fig.8:20) asignan al Altar G3 la fecha dedicatoria de 9.16.15.0.0 correspondiente al 19 de febrero de 766 .

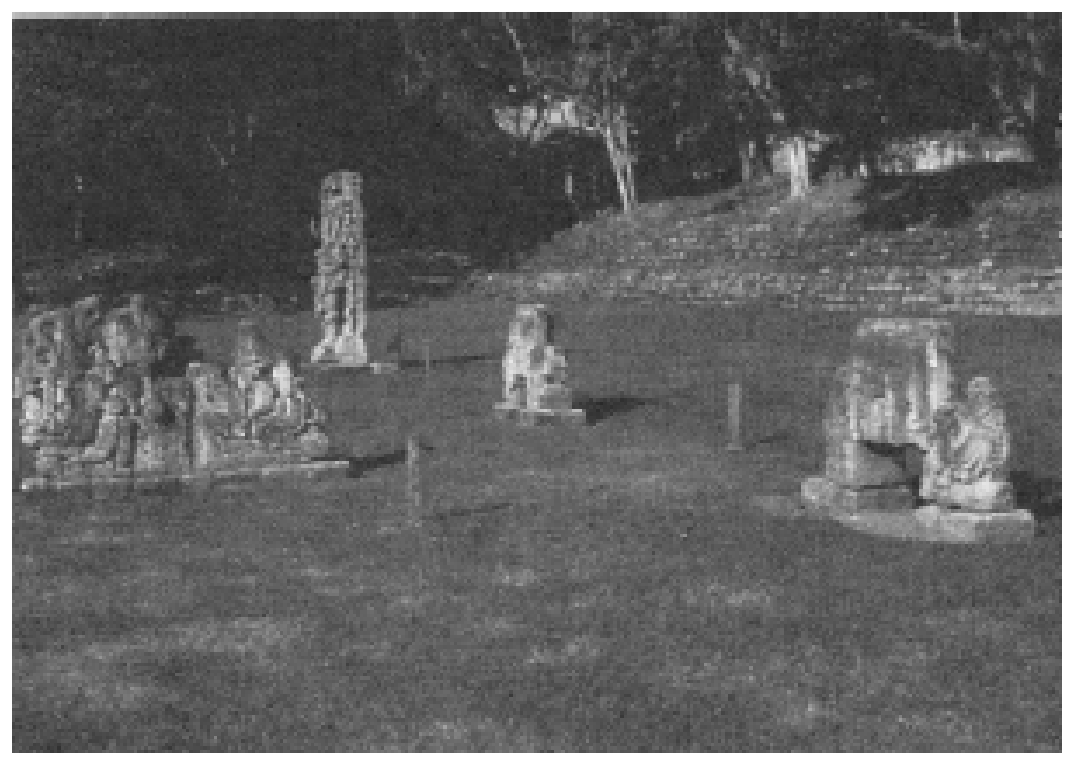

Figura 5. Los tres altares colocados por Yax-Pasaj, viendo hacia el noreste.

Fuente: Schele \& Mathews. (1998, pág. 170 Fig.4.37; Fotografía de Justin Kerr y Macduff Everton).

Con un enfoque arqueoastronómico, Pineda de Carías, Véliz y Agurcia Fasquelle encontraron que, el punto de intersección de los ejes de los altares $\mathrm{G}$, constituye un lugar de observación astronómica muy importante para los mayas de Copán. Desde ese lugar, viendo hacia el oeste a lo largo del año, sobre las Estelas A, 4, B y $\mathrm{C}$ se observa respectivamente la puesta del Sol en los días de los solsticios de invierno, equinoccios, pasos del Sol por el cenit y los solsticios de verano (Pineda de Carías, Véliz, \& Agurcia Fasquelle, 2002).

En el Manual de los Monumentos de Copán, Honduras, Biro y Reents-Budet (2010, págs. 195-207) atribuyen los Altares G a Yax Pasaj Chan Yopaat, Décimo Sexto 
Gobernante de Copán que reinó entre los años 763-822 D.C., conocido también como Yax Pac, Madrugada, Nuevo Sol en el Horizonte. La fecha de dedicación de cada altar es: 9.17.0.0.013 Ahau 18 Kumku (24 de enero del año 771 D.C.) para el Altar G3 (CPN 14); 9.18.5.0.0 4 Ahau 13 Keh (15 de septiembre del año 795 D.C.) para el Altar G2 (CPN 15); y 9.18.10.0.0 10 Ahau 8 Sak (19 de agosto del año 800 D.C.) para el Altar G1 (CPN 13).

En la actualidad, el Instituto Hondureño de Antropología e Historia, al documentar en el Parque Arqueológico de Copán los altares $\mathrm{G}$, lo hace en su conjunto, sin hacer distinción cuál es cada uno de los altares. En las Figura 6, 7 y 8 presentamos la disposición actual de los tres altares $\mathrm{G}$.

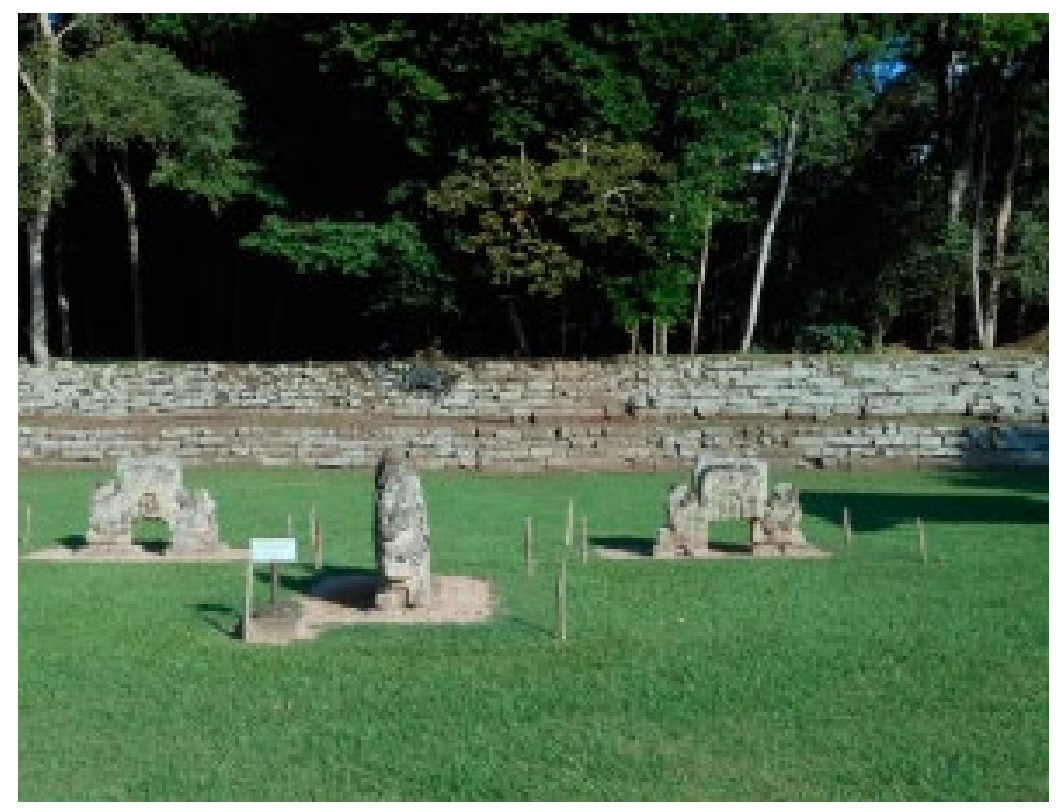

Figura 6. Vista hacia el este, de la situación actual de los tres altares G. Es evidente que solamente en el frente del Altar G1 aparece una placa. Fotografía: Ricardo Agurcia Fasquelle. 


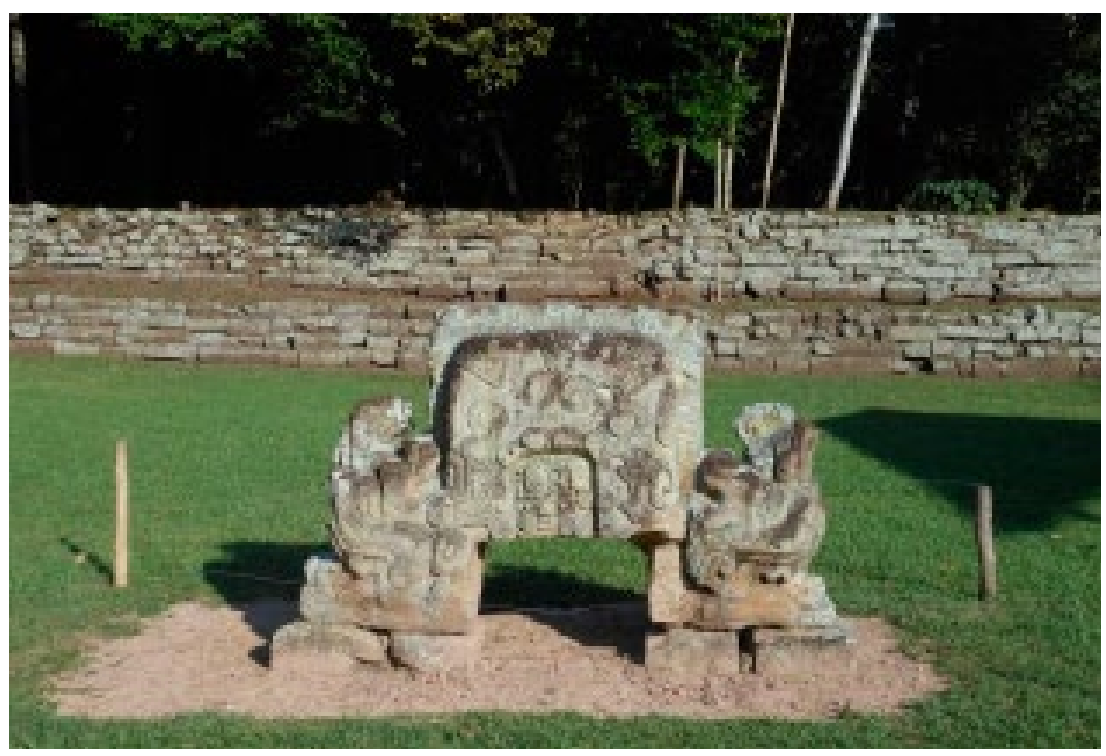

Figura 7. Vista hacia el este del Altar G2. Tal como se ve hoy día, sin placa. Fotografía: Ricardo Agurcia Fasquelle.

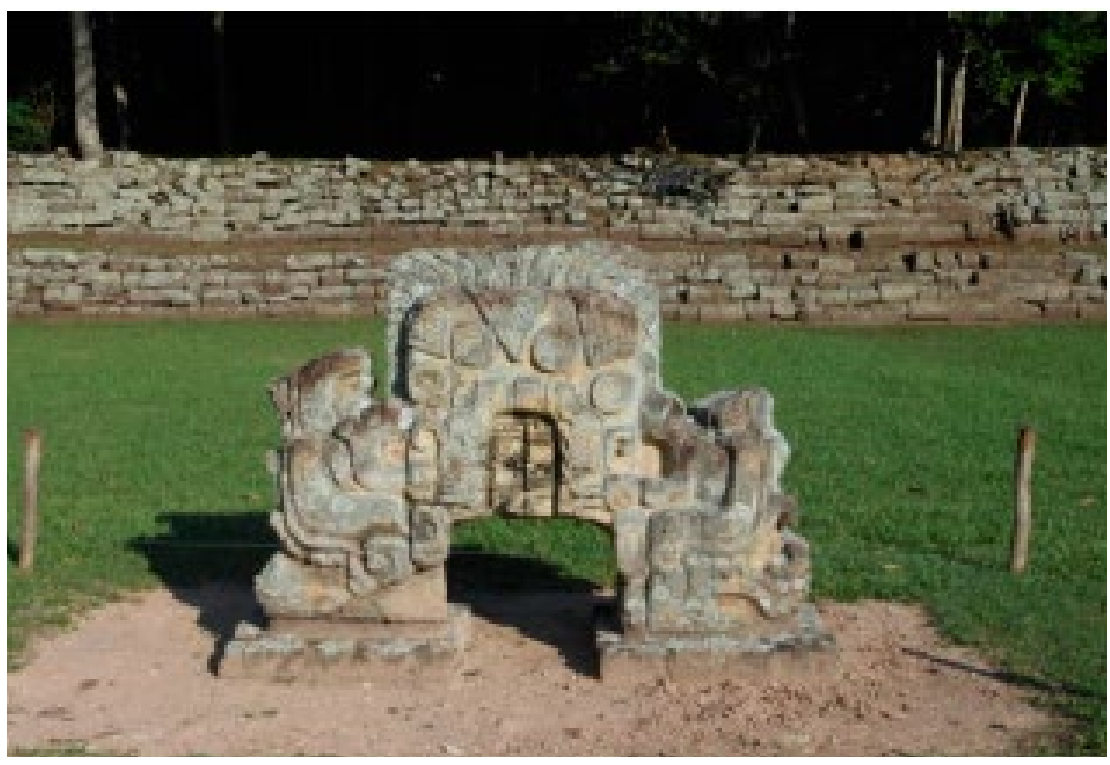

Figura 8. Vista hacia el este del Altar G3. Tal como se ve hoy día, sin placa. Fotografía: Ricardo Agurcia Fasquelle. 
El objetivo de este trabajo ha sido contestar las preguntas: ¿tienen los altares $\mathrm{G}$ la orientación longitudinal con que originalmente los colocaron los mayas? ¿Cuál es la orientación más probable? ¿Con qué intención fueron colocados los altares en el lugar que los encontramos? Con este propósito hicimos una revisión cuidadosa y comparativa de las diferentes anotaciones, referencias y descripciones realizadas por diferentes exploradores, particularmente arqueólogos, que a lo largo del tiempo han incluido en sus trabajos de campo e investigaciones a los altares G. También hicimos observaciones astronómicas de la puesta del Sol, situados en medio de los altares $\mathrm{G}(\mathrm{G} 1, \mathrm{G} 2, \mathrm{G} 3)$. Después de una revisión bibliográfica exhaustiva, seguida de una recopilación de evidencia gráfica y fotográfica pertinente, y de visitar in situ los altares localizados en el Parque Arqueológico de Copán, en este trabajo presentamos los resultados, un análisis minucioso de las diferentes orientaciones longitudinales encontradas para los altares G. La conclusión más importante a la que hemos llegado es que, basados en argumentos arqueoastronómicos, lo más probable es que la orientación actual de los altares $G(G 1, G 2$ y G3), fue la orientación original con que fueron colocados durante el reinado del Décimo Sexto Gobernante de Copán.

\section{DIFERENTES ORIENTACIONES DE LOS ALTARES G}

Los primeros datos de los altares $G$ revelados por Stephens, más allá de la existencia de un bulto de piedras de un altar caído, no dan ninguna pista acerca de la localización y orientación de cada uno de los altares G. Sin embargo, de las fotografías de Maudslay, sí es posible ver que dos de los altares están orientados en dirección este-oeste, y que el tercero está orientado en la dirección norte-sur. El altar más cercano a la Estela F es el Altar G3, orientado de la misma manera que el Altar G1 que está en el centro. Ambos altares en las dos fotografías de Maudslay, aparecen orientados en dirección este-oeste. El Altar G2, más cercano a la Estela $\mathrm{H}$, está orientado en dirección norte-sur, su orientación es diferente de la de los otros dos altares. Evidente pues resulta que, los tres altares localizados entre la estela $\mathrm{F}$ en el extremo norte y la Estela $\mathrm{H}$ en el extremo sur, no tienen la misma orientación. Llama la atención que, en ambas fotografías, en el extremo este del Altar G3 aparece colocado un bulto de piedras, quedando la duda si tal conjunto, sirvió temporalmente para mantener levantado el Altar para luego colocarlo en otra orientación. 
Al describir la orientación de los tres altares $G$, Baudez dice que todos están orientados hacia el oeste, aunque esta expresión dentro del contexto se refiere más bien hacia donde están orientadas los monumentos alineados hacia el este: CPN 16 (Estela H), CPN 13 (Altar G1), CPN 14 (Altar G2), CPN 15 (Altar G3), CPN 11 (Estela F). Él dice que su localización sugiere que ellos fueron colocados como una contraparte de CPN 43 (Estela 4). (Baudez, 1994, pág. 55).

Posteriormente, en la fotografía presentada por Schele y Mathews, y sobre lo que nosotros mismos hemos podido comprobar in situ, la orientación actual de los tres altares es, en dirección norte-sur para los Altares G2 y G3, cercanos respectivamente a la Estela $\mathrm{H}$ y a la Estela $\mathrm{F}$, y la dirección este-oeste para el Altar G1 localizado ligeramente en medio y enfrente de los otros dos altares G2 y G3 y de las dos estelas $\mathrm{H}$ y $\mathrm{F}$.

Como un resultado de las diferentes orientaciones encontradas, en la Figura 9 incluimos un diagrama que muestra las orientaciones atribuidas a los Altares $\mathrm{G} 1$, G2 y G3. 


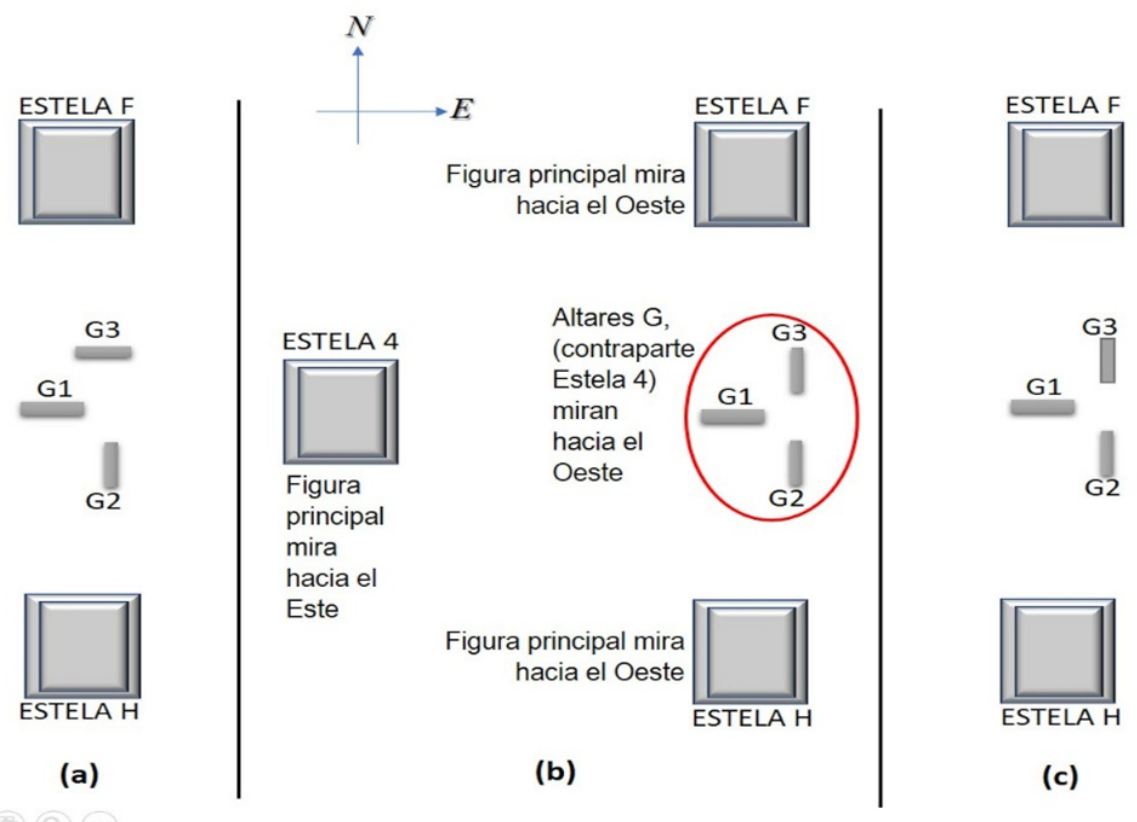

Figura 9. Diagrama que muestra diferentes orientaciones atribuidas a los Altares G.

(a) En el lado izquierdo, según fotografías de Maudslay (1898-1902). (b) En la parte central, según texto de Baudez (1994). (c) En el lado derecho, según fotografía de Schele y Mathews (1999) y situación actual.

\section{DISCUSIÓN Y CONCLUSIONES}

Por ser la referencia más temprana en la literatura, se supone que las ubicaciones de Maudslay están más cercanas a la realidad, más apegadas a la ubicación original que los mayas seleccionaron para estos altares. Según sus fotos y sus referencias a fechas, Maudslay (1898-1902) claramente señala que el Altar G2 está al sur y tiene la fecha, en Rueda Calendárica, 4 Ajáu 13 Ceh (Plates 52c, 53a, 54a, 116). Comenzando con el $\mathrm{G} 1$ y yendo de la fecha más reciente a la más antigua y en dirección opuesta a las manecillas del reloj, Maudslay pasa al sur, al G2, y luego al norte, al G3. Aunque fue Strömsvik (1946, pág. 36) quien dejó estos altares en su actual posición, ubicación y orientación, en su texto no menciona en qué dirección está cada altar. Además, en el mapa que aparece después de la página 83 (Strömsvik, 1946), sólo aparecen rayitas para los altares, sin los números. Pero Baudez (1994, págs. 7, Figura 1) y Fash y Agurcia (2007, págs. 30, Figura 4) 
ubican el Altar G2 al norte y el G3 al sur, asignando a cada denominación su fecha respectiva, de acuerdo con la placa, al Altar G2 el año 795 d.C. y al Altar G3 el año 766 d.C. Éste, seguramente, ha sido el patrón que, inadvertidamente, todos hemos seguido. Nadie había notado el detalle de que las plaquitas estaban cambiadas, y que en el Manual de los Monumentos de Copán (Agurcia Fasquelle \& Veliz, 2010) ya se ha aclarado.

Después de muchas consultas y reflexiones, hemos llegado a la conclusión de que lo que ha sucedido es lo siguiente. Cuando el IHAH elaboró y colocó las plaquitas, los técnicos se confundieron y las colocaron a la inversa. Simplemente intercambiaron las plaquitas de los altares G2 y G3. Los investigadores y los escritores posteriores no se percataron de esta situación y se ha continuado con el error, sin darse cuenta de que las fechas no concuerdan con los altares in situ. Aunque uno de ellos no está con la misma orientación, tal como se ha explicado, los altares, comprobado recientemente por las fechas, continúan en la misma ubicación: el Altar G2 (766 d.C.) al sur y el G3 (795 d.C.) al norte.

Esta situación anómala la corrigió el IHAH por medio de la sencilla acción de cambiar las plaquitas, poniendo la del Altar G2 al sur y la del Altar G3 al norte, y teniendo en cuenta que se tendrá que intercambiar las fechas que muchos autores hemos venido presentando en nuestros escritos para estos dos altares. Aunque hoy día el IHAH, por alguna razón, ha removido las placas de los Altares G2 y G3 dejando únicamente la del Altar G1 (Figura 6).

El otro detalle es que, de acuerdo con las fotografías de Maudslay (Figuras 1 y 2), el Altar G3, al norte, estaba orientado de este a oeste, igual que el G1. No obstante, ningún investigador hace mención de cambios (Maudslay, 1898-1902; Morley, 1920; Strömsvik, 1946; Longyear, 1952). Hoy día este altar aparece orientado de norte a sur (Figura 6).

Este último cambio implica serias consecuencias para los resultados de las investigaciones arqueoastronómicas. Si la orientación del Altar G3 era distinta, fue porque los mayas copanecos deseaban hacer referencia a un evento 0 a un acontecimiento distinto. Bajo las circunstancias de cambios en orientación y muy posiblemente en ubicación, parecería que nunca llegaremos a saber con exactitud cuál fue la motivación de los mayas al colocar este altar con la orientación y en el punto en que lo hicieron. Sin embargo, los estudios arqueoastronómicos nos presentan otras evidencias que nos pueden llegar a apuntar cuál pudo haber sido la orientación original de los altares. 
Las Estelas $\mathrm{F}$ y $\mathrm{H}$ definen una línea norte-sur en el lado este de la Plaza. En medio de estas estelas, desde el punto que Pineda de Carías, Véliz y Agurcia Fasquelle han llamado el punto $G$, en una dirección perpendicular hacia la Estela 4 se marca la línea este-oeste. La localización de los altares $\mathrm{G}$, tal como lo dice Baudez, es la contraparte de la Estela 4, localizada en el lado oeste de la Gran Plaza. Parece entonces razonable resguardar este punto de observación privilegiado, localizando con sus ejes longitudinales en la dirección norte-sur con los altares $\mathrm{G} 3$, dirigido hacia la Estela F, y el Altar G2, dirigido hacia la Estela $H$. El Altar G1, en dirección perpendicular este-oeste, sobresaliendo sobre los otros dos altares y de las estelas $\mathrm{F} \mathrm{y} \mathrm{H}$, define de mejor manera la línea este-oeste que lo une hacia la Estela 4.

Esta es la argumentación por la que principalmente creemos que la orientación actual en que se encuentran localizados los altares $G(G 1, G 2, G 3)$, es la que probablemente utilizaron los mayas durante el reinado del Décimo Sexto Gobernante de Copán. Una posición que efectivamente se ajustaba al Plan del Décimo Tercer Gobernante en su Plan de remodelación de la Plaza Principal, y la demarcación de un punto privilegiado de observación que seguramente sirvió a los mayas de Copán para marcar sus registros del tiempo y a partir de ello los calendarios.

Agradecimientos. Agradecemos a los profesores del Departamento de Arqueoastronomía y Astronomía Cultural de la Facultad de Ciencias Espaciales de la Universidad Nacional Autónoma de Honduras, y a la Asociación Copán, por el apoyo brindado para realizar los viajes de campo y obtener información y datos en Copán. 


\section{BIBLIOGRAFÍA}

- Biro, Peter \& Dorie Reents-Budet (2010) Los Monumentos de la Plaza Principal en Agurcia Fasquelle, R., \& Veliz, V. (Edits.). (2010). Manual de los Monumentos de Copán, Honduras. Recuperado el 25 de Noviembre de 2015, de FAMSI Fundación para el avance de los Estudios Mesoamericanos, Inc.: http://www. famsi.org/spanish/research/copan/monuments/index.html

- Baudez, C. F. (1994). Maya Sculpture of Copan: The Iconography. Norman: University of Oklahoma Press.

- Fash, W., \& Agurcia, R. (2007). History Carved in Stone: A Guide to the Archaeological Park of the Ruins of Copan. San Pedro Sula.

- Longyear, J. M. (1952). A Study of Southeastern Maya Pottery (Vol. Publication No. 597). Washington D.C.: Carnegie Institution of Washington.

- Maudslay, A. P. (1898-1902). Archaeology. En A. P. Maudslay, Biologia CentraliAmericana. Londres: Porter and Dulau.

- Morley, S. G. (1920). The Inscriptions at Copan (Vol. Publication No. 219). Washington D.C.: Carnegie Institution of Washington.

- Pineda de Carías, M. C., Véliz, V., \& Agurcia Fasquelle, R. (2002). Acerca de las observaciones del Sol realizadas en la Gran Plaza del Parque Arqueológico de Copán Ruinas, Honduras. Yaxkin, XXI, 15-44.

- Schele, L., \& Freidel, D. (1990). A Forest of Kings: The Untold Story of the Ancient Maya. New York: William Morrow.

- Schele, L., \& Mathews, P. (1998). The Code of Kings. The Language of Seven Sacred Maya Temples and Tombs. New York: A Touchstone Book. Simon \& Schuster.

- Stephens, J. L. (1969). Incidents of Travel in Central America, Chiapas, and Yucatan (Primera Edición en 1841 Arthur Hall, Virtue and Co. Londres ed., Vol. I y II). Dover Publications.

- Strömsvik, G. (1946). Guía de las Ruinas de Copán. Tegucigalpa: Talleres TipoLitográficos "Aristón". 